\title{
Research and Implementation of a WPT System Applied in High Voltage Environment
}

\author{
Like $\mathrm{Gao}^{1, \mathrm{a}}$, Xingran $\mathrm{Gao}^{2, \mathrm{~b}^{*}}$ and Hong Zhou ${ }^{3, \mathrm{c}}$ \\ ${ }^{1}$ Guangxi Electric Power Research Institute, Nanning 530023, China \\ ${ }^{2}$ Wuhan University, Wuhan 430072, China \\ ${ }^{3}$ Wuhan University, Wuhan 430072, China \\ agao_lk.sy@gx.csg.cn, bgaoxran@foxmail.com, chzhouwuhee@whu.edu.cn
}

\begin{abstract}
Keywords: two-coil, wireless power transfer, inductive coupling, high voltage Abstract. This paper provides a new scheme for wireless power transfer (WPT) technology based on magnetically coupled resonance. The technology is used to solve the problem of reliable power supply for the monitoring terminals of high voltage power grid (HVPG). Because the technology takes the magnetic field as the medium, it can transmit power from high potential side to low potential side without destroying the insulation condition of high voltage line. In this paper, a WPT system under high voltage environment is designed, and has high transmission efficiency in mid-range distance. The availability of this method is verified by a set of experimental platforms.
\end{abstract}

\section{Introduction}

Grid monitoring terminals in high-voltage and extra-high voltage environment is of particular importance to ensure the stability and security of power system ${ }^{[1-2]}$. However, reliable power supply for monitoring terminals of HVPG is always the key problem to limit the intelligent monitoring and management of power system ${ }^{[3]}$. Most of the traditional solutions use distributed energy such as wind energy, solar energy, etc., and the inherent instability of distributed energy makes the terminals not get reliable energy supply, especially in southern China where rainy weather is frequent ${ }^{[4]}$. However, a prominent contradiction forms. Although there is plenty of electric energy on the high voltage transmission line, it cannot be directly used from the high potential side due to insulation requirements.

In recent years, the rapid development of WPT technology makes it possible to transfer power directly from high potential side to low potential side, which provides a new possibility for the power supply for intelligent monitoring terminals. WPT was first proposed by Nekola Tesla, the father of WPT, a century ago ${ }^{[5]}$, and has been an active research topic since MIT reported their achievements in $2007^{[6]}$. The results greatly promoted the application of WPT in $\mathrm{EV}^{[7-8]}$, mobile device ${ }^{[9-10]}$, medical implants ${ }^{[11-12]}$ and so on. Characterized by mid-range and high efficiency ${ }^{[13]}$, WPT technology can be potentially applied in the field of smart grid.

This paper designs a set of WPT system applied in high voltage environment as shown in Fig. 1, and its working principles are analyzed. Meanwhile, the performance of the system is verified by simulation and related experiments. The aim is to get a complete system design and operation scheme, in order to promote the application of WPT in smart grid.

\section{System Structure and its Modeling Analysis}

System overall structure. According to the structure as shown in Fig. 1, the system model is shown in Fig. 2. $S_{l}$ is a frequency adjustable AC voltage source; $L_{1}, C_{1}$ compose the resonator of transmitting side and $R_{S}$ is the internal resistance of transmitting side; $L_{2}, C_{2}$ compose the resonator of receiving side and $R_{W}$ is the internal resistance of receiving side; $R_{L}$ is equivalent resistance of load. WPT can be realized by the two magnetically coupled resonators when driven by a suitable source.

As shown in Fig. 2, both the working current at the transmitting side and the receiving side can be obtained according to Kirchhoff's law of voltage, as shown in the Eq. 1 and Eq. $2^{[14-15]} . Z_{1}$ and $Z_{2}$ are 
the impedance of transmitting side and receiving side, respectively. $M$ is the mutual inductance between the two coils and $\omega$ is the angle frequency of the driving power source.

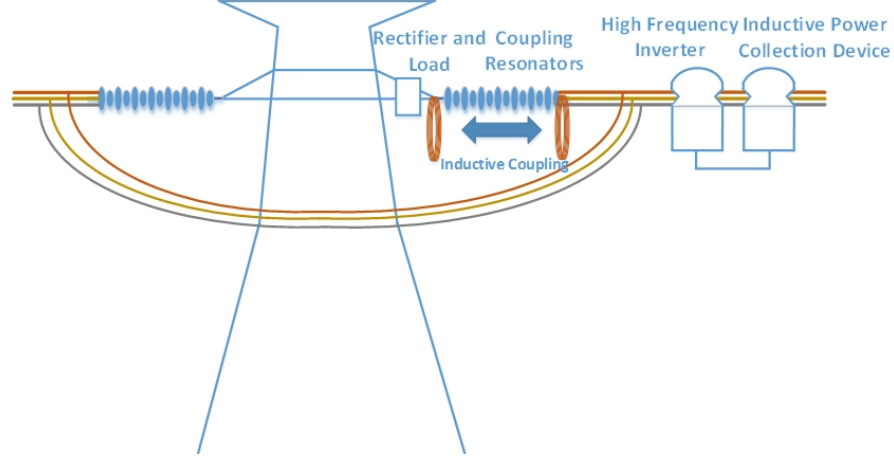

Fig. 1. System installation structure diagram

$$
\begin{aligned}
& I_{1}=\frac{Z_{2}}{Z_{1} Z_{2}+\omega^{2} M^{2}} V_{s} \\
& I_{2}=\frac{j \omega M}{Z_{1} Z_{2}+\omega^{2} M^{2}} V_{s}=\frac{j \omega M}{Z_{2}} I_{1}
\end{aligned}
$$

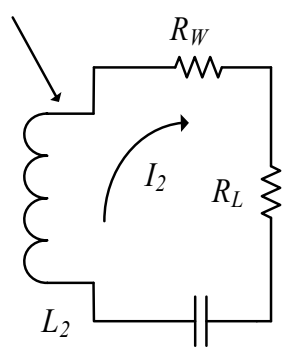

Fig. 2. Equivalent circuit diagram

By releasing the Ohm's law in complex frequency domain, the WPT model can be simplified as shown in Fig. 3. $Z_{r e}$ in Fig. 3(a) is the reflected impedance by receiving side according to conventional transformer theory as shown in Eq. 3; $j \omega M I_{l}$ in Fig. 3(b) is the induced electromotive force at receiving side according to Faraday's law of induction. Relative calculation shows that the power consumption of $Z_{r e}$ is conjugated with the power consumption of the receiver as shown in Eq. 4. Since reactive power does not consume energy, ratio between the real part of $Z_{r e}$ and the overall resistance in Fig.3-a can be viewed as the transmission efficiency.

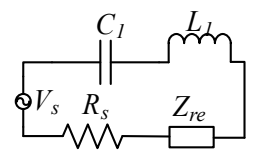

(a)

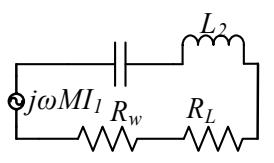

(b)

Fig. 3. Simplified model of a two-coil WPT system

$$
\begin{aligned}
& Z_{r e}=\frac{\omega^{2} M^{2}}{R_{W}+R_{L}+j\left(\omega L-\frac{1}{\omega C}\right)} \\
& \left|I_{1}\right|^{2} Z_{r e}=\left(\left|I_{2}\right|^{2} Z_{2}\right)^{*}
\end{aligned}
$$

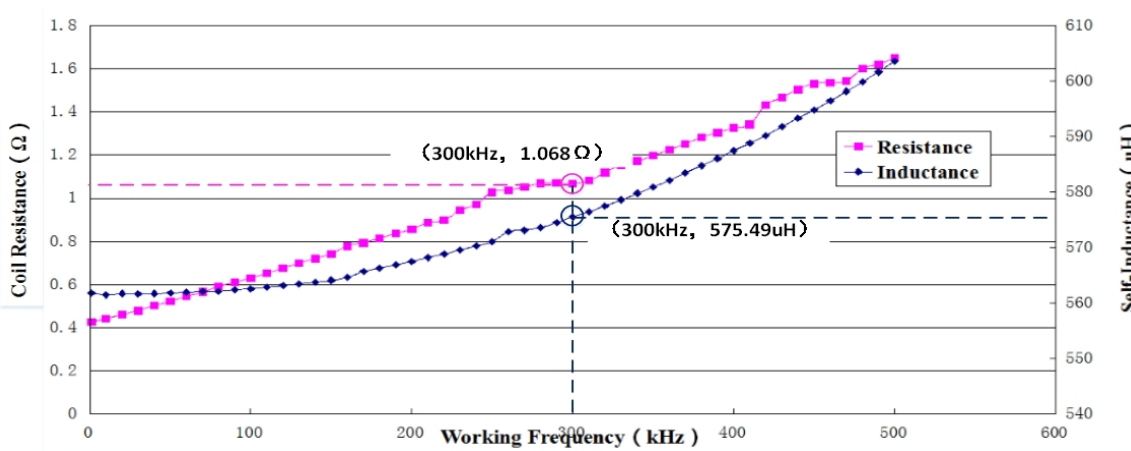

Fig. 4. Measurement results of coil inductance/resistance against working frequency

Resonant circuit design. The transmission efficiency is calculated as shown in Eq. 5. Under the the invariant conditions including resistance $R_{S}$ and $R_{W}$ and the load $R_{L}$ and mutual inductance $M$, it only needs to adjust the operating frequency to make the real part of the $Z_{r e}$ obtain the maximum value to obtain the maximum transmission efficiency. The derivation of impedance $Z_{r e}$ on angular frequency $\omega$ can obtain Eq. 6.

$$
\begin{aligned}
& \eta=\frac{\left|I_{2}\right|^{2} R_{L}}{\left|I_{1}\right|^{2} R_{s}+\left|I_{2}\right|^{2}\left(R_{L}+R_{W}\right)}=\frac{\operatorname{Re}\left(Z_{r e}\right)}{R_{s}+\operatorname{Re}\left(Z_{r e}\right)} \cdot \frac{R_{L}}{R_{W}+R_{L}} \\
& \omega=\frac{1}{C_{2} \sqrt{\left(\frac{L_{2}}{C_{2}}-\frac{1}{2}\left(R_{2}+R_{L}\right)^{2}\right)}} \approx \frac{1}{\sqrt{L_{2} C_{2}}}
\end{aligned}
$$


From Eq. 6 we can know that when system works at the natural angular frequency of $\omega_{2}$ of the receiving side, the WPT system can obtain the maximum transmission efficiency. This is because the receiving side has the largest power factor in $\omega_{2}$, and the maximum reflected impedance is generated at the transmitting side, as shown in Eq. 7.

$$
Z_{r e}=\frac{\omega^{2} M^{2}}{R_{W}+R_{L}}
$$

According to Eq. 7, the system maximum transmission efficiency is positively related to mutual inductance $M$ and working frequency $\omega$, and negatively correlated with internal resistance $R_{S}$ and $R_{W}$. Among them, $R_{S}$ and $R_{W}$ are also positively related to working frequency due to skin effect.

For a WPT system on the high voltage line, the transmission distance must be longer than the insulation distance required by the line. With the increase of voltage level, the insulation distance also increases; however, the coupling coefficient between the coils can be quickly reduced by $k$. Therefore, the designed system needs to maintain a high transmission efficiency in a large transmission distance (e.g. $1.1 \mathrm{~m}$ for $110 \mathrm{kV}$ ), thus ensuring enough received power at low potential side. Because the coupling coefficient $k$ can be quite attenuate in a long distance, the mutual inductance $\mathrm{M}$ is the main factor to limit the transmission efficiency. Therefore, in the design of the WPT system, a appropriate size of the coil should be designed according to the actual situation, so as to ensure a certain mutual inductance. Then, the transmission efficiency is achieved as high as possible by the trade-off between the internal resistance and the natural frequency.

To obtain a large mutual inductance, a multi-turn cylindrical coil is used. Taking into account the insulation distance and the installation condition of the $110 \mathrm{kV}$ line, the designed size of the coil is of $40 \mathrm{~cm}$ diameter and $6 \mathrm{~cm}$ height. At the same time, $0.004 * 1200$ Litz wire is used to reduce the internal resistance caused by skin effect, thus increasing $Q$ value ${ }^{[16]}$. The relationship between coil inductance and resistance against working frequency is shown in Fig. 4. The coupling coefficient $k$ is 0.00356 by the elliptic integral when the working distance is fixed at $1.1 \mathrm{~m}$.

When considering the main factors affecting the transmission efficiency of the system, the heat loss of MOSFET is also a large proportion, which can not only reduce the system efficiency, but also increase the risk of damage to the equipment. Heat loss calculation formula of Mosfets is shown as Eq. 8, where $P_{1}$ is the conduction loss and $P_{2}$ is the switch loss of one single switch. Eq. 8 is obtained under the condition of $48 \mathrm{~V}$ DC voltage, the average power $100 \mathrm{~W}$ of a half bridge driver. Combining Eq. 5 and Eq. 8, an expression of the maximum transmission efficiency of the resonant system with frequency variation is shown as Eq. 9 .

$$
\begin{aligned}
& P_{\text {mos }}=P_{1}+f \cdot P_{2}=0.92+f \cdot 0.0000524 \\
& \eta=\frac{\frac{\omega^{2} M^{2}}{R_{1}+1+R_{L}}}{R_{1}+0.1+\frac{\omega^{2} M^{2}}{R_{1}+1+R_{L}}} \cdot \frac{R_{L}}{R_{1}+1+R_{L}} \cdot \frac{100-P_{\text {mos }}}{100}
\end{aligned}
$$

In Eq. 9, the default frequency is the natural frequency of the receiving side. $R_{1}$ is the measured coil resistance in different frequency, and $0.1 \Omega$ is the approximate resistance in transmitter's capacitor and other parts ; $1 \Omega$ is the approximate resistance in receiver's capacitor and rectifier and $R_{L}$ is taken $6.6 \Omega$ as the equivalent resistance of online monitoring device. As shown in Fig. 5, system can obtain a high transmission efficiency in the vicinity of $400 \mathrm{kHz}$.Considering the reliability of the inverter circuit in the case of heat dissipation, a $460 \mathrm{pF}$ resonant capacitor is selected, which makes the natural frequency of the system be in the vicinity of $300 \mathrm{kHz}$. 


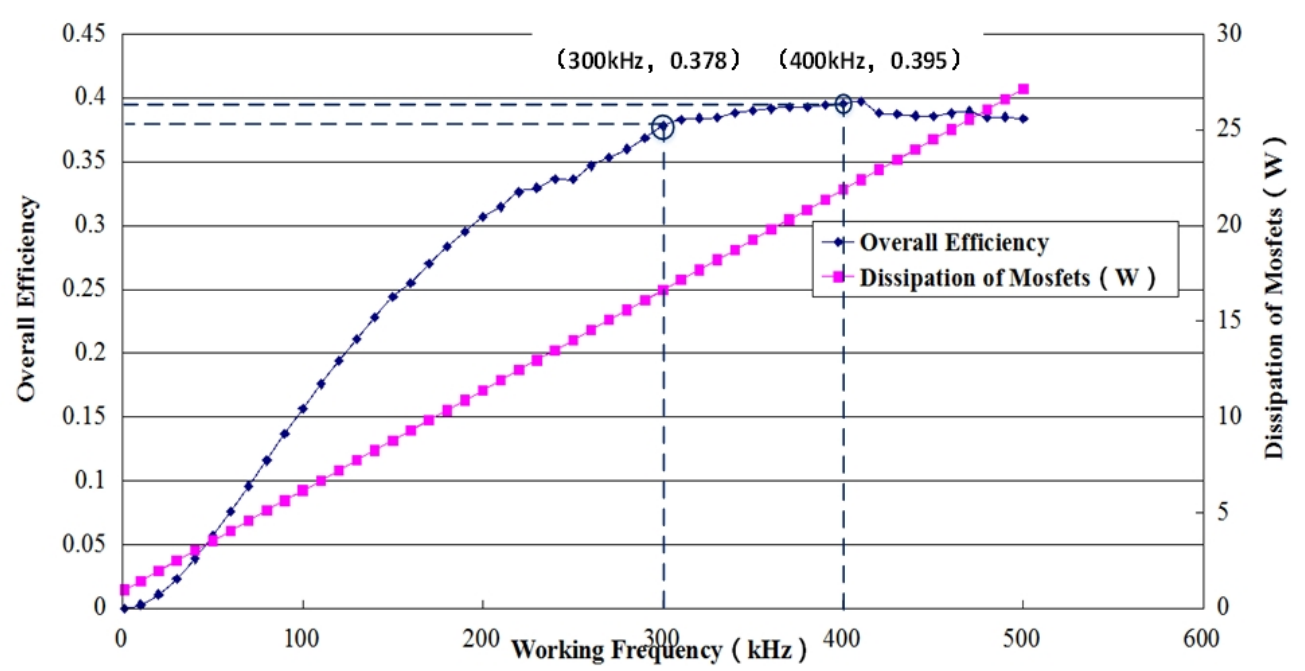

Fig. 5. Mosfets dissipation and system efficiency against working frequency

System operating frequency selection. The WPT system on the high voltage line is designed to provide sufficient power for the low potential side. However, the maximum power frequency is often inconsistent with the maximum efficiency frequency, which is particularly evident in over-coupling cases. So it is important to analyze the characteristics of the output power, and select a appropriate operating frequency to adapt to the specific conditions of the high voltage line.

As shown in Fig. 6, when system works in the under-coupling condition, the output power at the the frequency of maximum transmission efficiency $\left(P_{1}\right)$, maximum transmitted power $\left(P_{2}\right)$ and maximum received power $\left(P_{3}\right)$ are nearly same. When system is in over-coupling condition, $P_{1}$ falls off quickly due to frequency splitting ${ }^{[16]}$ and $P_{2}$ is slightly smaller than $P_{3}$. Considering the harsh electromagnetic environment of the high voltage side, the control circuits at Tx side have to be shielded by a specialized metal box and communication links between Tx side and Rx side can hardly be established, which means Tx side cannot get the information of Rx side. Since the frequency of maximum transmitted power point can be detected with the information of Tx side only, it is selected as the operating frequency of the WPT system.

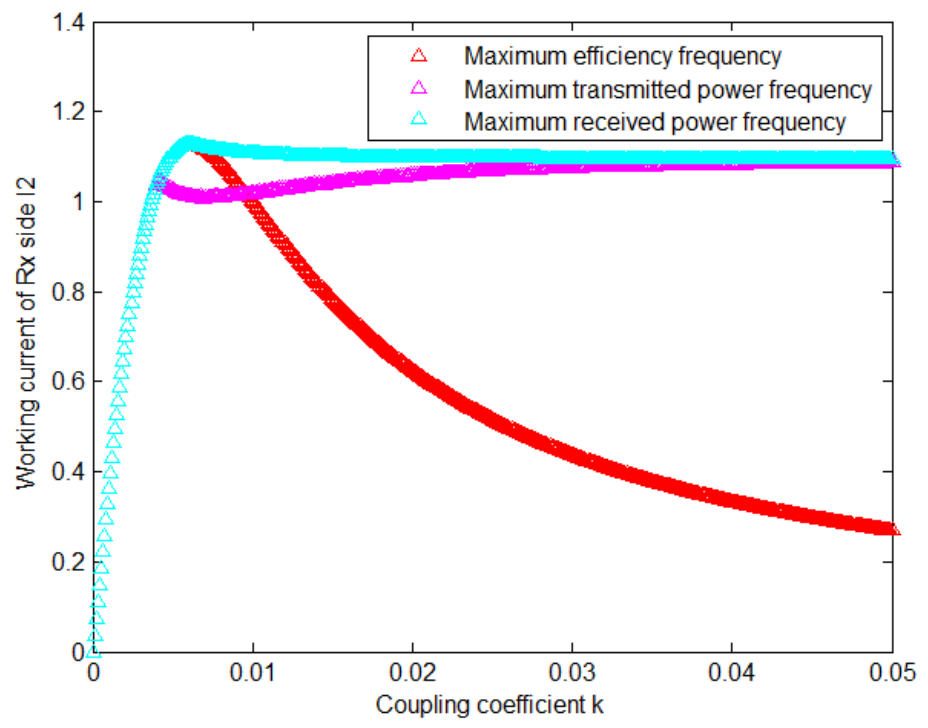

Fig. 6. Working current of $\operatorname{Rx} \operatorname{side}\left(I_{2}\right)$ against coupling coefficient $k$ in different operating frequency

\section{System Implementation}

The implementation of the system under high voltage environment is shown in Fig. 7. In Fig. 7, the high-voltage inductive power collection device can harvest $\mathrm{AC}$ power of $50 \mathrm{~Hz}$ from the simulated large current line, then converts the AC power to DC power of $48 \mathrm{~V}$ and supply it to the high frequency inverter. The high frequency inverter part is shown in Fig. 8, which includes a signal generator and an output circuit. In the signal generator part, a DDS module is used to obtain a square 
wave signal with adjustable frequency and duty ratio. Then the signal, processed by the driving circuit, drives a inverter bridge composed of Mosfet chips, thus realizing the $48 \mathrm{~V} \mathrm{DC}$ inverter. The inverter circuit generates $24 \mathrm{~V}$ AC voltage by a class-D half bridge, and it also has a hall sensor, which can detect the operating current of the system in real time. The measured results are used to detect the maximum transmitted power frequency as the operating frequency of the system, and also to adjust the duty ratio of the driving signal to ensure that the output power of the system is no more than the maximum output power allowed by the current on line.

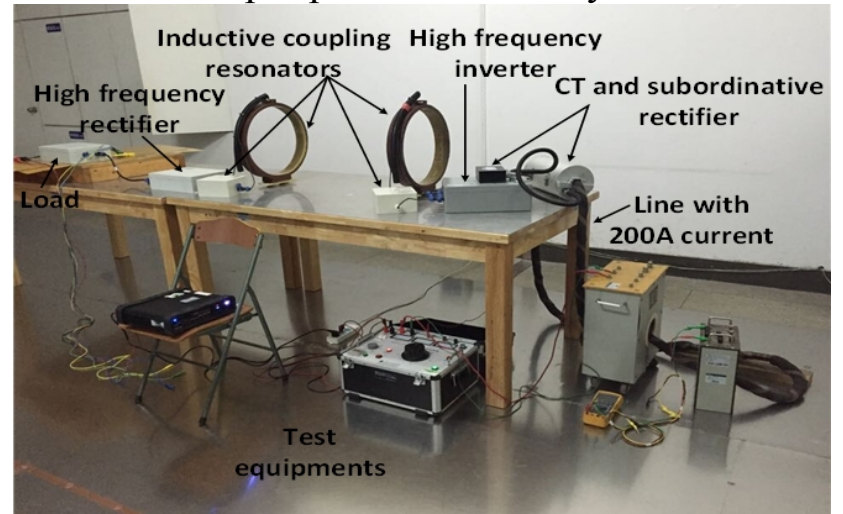

Fig. 7. Implementation of the WPT system applied in high voltage environment

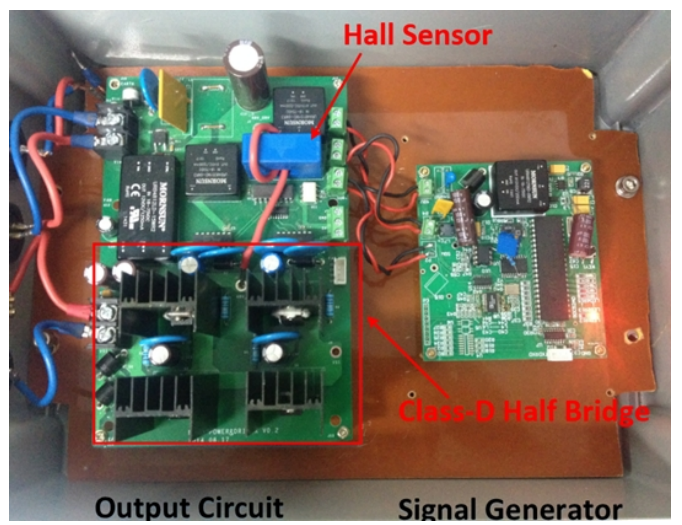

Fig.8. PCB design for Tx side

The two coils of $0.4 \mathrm{~m}$ diameter and 28 turns are used as the magnetically coupled resonators, and the outer layer is sealed by epoxy resin to ensure the waterproof property. Capacitors with the advantages of small resistance and high withstand voltage are connected in series with the coils and they can meet the requirement of high transmission efficiency and high resonant voltage.

The received power is still of high frequency and cannot be used for common electronic equipment. Therefore, a rectifier and filter circuit are added at the receiving side to obtain a stable DC power. A wide input power module is also used to provide the standard $12 \mathrm{~V} \mathrm{DC}$ power. In addition, in order to ensure the short-term endurance of load equipment under the condition of line outage, a super capacitor module is connected in parallel with the power input of the load.

When measuring the transmitted power and transmission efficiency of the WPT system, a resistive load of $6.6 \Omega$ is used as an alternative of the nonlinear load in Fig. 7. Under the condition of 200A current on line, the distance between the Tx side coil and Rx side is adjusted from the $30 \mathrm{~cm}$ to $120 \mathrm{~cm}$ at interval of $10 \mathrm{~cm}$. At various intervals, the operating frequency is automatically adjusted to the maximum transmitted power frequency, and the corresponding transmitted power, received power and transmission efficiency are shown in Fig. 9.

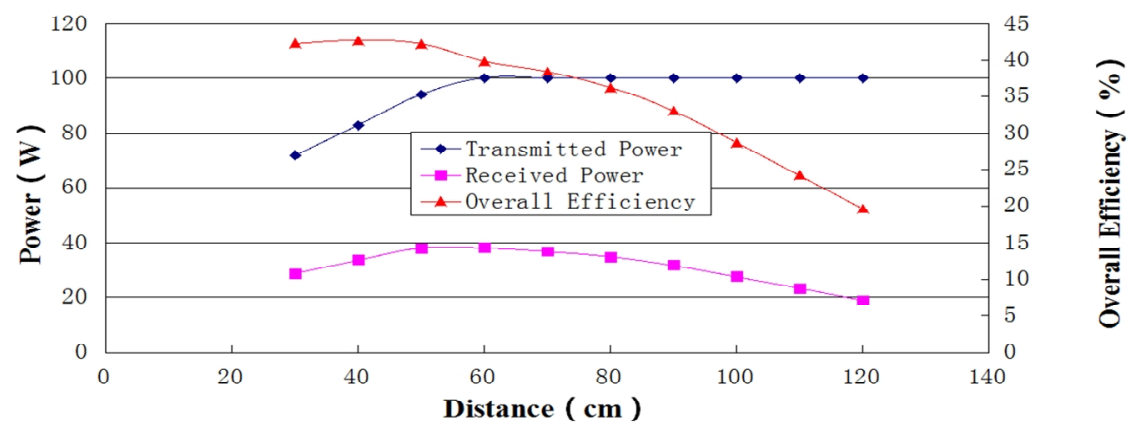

Fig. 9. Transmission efficiency in different distance

As can be seen from Fig. 9, in the case of no more than $50 \mathrm{~cm}$ distance, the maximum transmitted power of the system is less than $100 \mathrm{~W}$ and is used as the transmitted power. In the case of no less than $60 \mathrm{~cm}$, The maximum transmitted power of the system is more than $100 \mathrm{~W}$. Due to the limitation of the output power of the inductive power collector, the transmitted power is automatically adjusted to be approx 100W. In Fig. 9, the expression of the received power / (transmitted power-6W) is adopted in the calculation of the transmission efficiency, where $6 \mathrm{~W}$ is the energy consumed by the control 
circuits at Tx side. As shown in Fig.9, in no more than $1.1 \mathrm{~m}$, the received power by load is above $20 \mathrm{~W}$, the transmission efficiency is more than 20\%. Therefore, the WPT system can provide sufficient power supply for most of the smart grid monitoring terminals at the required insulation distance.

\section{Conclusion and Prospect}

This paper presents the design and implementation of a WPT system applied in high voltage environment. The maximum transmission efficiency is analyzed and design principles are proposed. For the practical system, more than $20 \mathrm{~W}$ power can be provided for Rx side in the condition of $1.1 \mathrm{~m}$ distance and 200A current on line. This technology can provide adequate and reliable power supply for most monitoring terminals, thus promoting the security of power system and reducing labor costs.

In future work, a set of WPT system of different size will be developed for lines of different voltage level to facilitate the application of WPT technology in smart grid field.

\section{Acknowledgment}

This work is supported by the National Natural Science Foundation of China (61374064) and China Southern Power Grid Science and Technology Project (K-GX2012-034). The authors would like to thank for that.

\section{References}

[1] Gungor V, Sahin S, Kocak T, et al., A survey on smart grid potential applications and communication requirements[J].IEEE Trans on Industrial Informatics, 2013, 9(1): 28-42.

[2] Yue Z M, Distribution networks in China under the smart grids environment[J]. IEEE Trans on Power Delivery, 2011, 26(1): 173-180.

[3] Moser M, Zangl H, and Bretterklieber T, An autonomous sensor system for monitoring of high voltage overhead power supply lines, e \& i Elektrotechnik und Informationstechnik, vol. 126, no.5, pp. 214-219, 2009.

[4] Zhao Xinming, Keutel T, Baldauf M, et al., Energy harvesting for a wireless-monitoring system of overhead hig-voltage power lines[J]. IET Generation Transmission \& Distribution, 2013, 7(2): 101-107.

[5] Tesla N, Apparatus for transmitting electrical energy: US 1119732[P]. 1914-10.

[6] Kurs A, Karalis A, Moffatt R, et al., Wireless power transfer via strongly coupled magnetic resonances[J]. Science, 2007, 317(5834): 83-86.

[7] Boys J T, Covic G A, Pick-up transformer for ICPT applications[C]. Electronics Letters. 2002:1276-1278.

[8] U.K. Madawala and D. J. Thrimawithana, A bidirectional inductive power interface for electric vehicles in V2G systems[J], IEEE Trans. Ind. Electron., vol.58, no.10, pp.4789-4796, Oct. 2011.

[9] K. Chang-Gyun, S. Dong-Hyun, Y. Jung-Sik, P. Jong-Hu, and B. H. Cho, Design of a contactless battery charger for cellular phone[J], IEEE Trans. Ind. Electron., vol. 48, no. 6, pp. 1238 - 1247, Dec. 2001.

[10] H. Jabbar, Y. S. Song, and T. T. Jeong, RF energy harvesting system and circuits for charging of mobile devices[J], IEEE Trans. Consum. Electron., vol. 56, no. 1, pp. 247 - 253, Feb. 2010.

[11] T. Sai Chun, F. A. Jolesz, and G. T. Clement, A wireless batteryless deep-seated implantable ultrasonic pulser-receiver powered by magnetic coupling[J], IEEE Trans. Ultrason., Terroelectr., Freq. Control, vol.58, no.6, pp.1211-1221, Jun. 2011. 
[12] F. Zhang, L. Xiaoyu, S. A. Hackworth, R. J. Sclabassi, and S. Mingui, In vitro and in vivo studies on wireless powering of medical sensors and implantable devices[J], in Proc. IEEE/NIH LiSSA, Apr. 9/10, 2009, pp.84-87.

[13] Xueliang Huang, Linlin Tan, et al., Review and research progress on wireless power transfer technology, Transactions of China Electrotechnical Society[J], vol.28, no.10, pp: 1-11, Oct. 2013.

[14] Hong Zhou, Bin Zhu, et al., Modelling and practical implementation of 2-coil wireless power transfer systems[J], Electrical and Computer Engineering, vol.2014, no.27, pp.1-8.

[15] Wenshan Hu, Hong Zhou, et al., Optimization algorithm and practical implementation for 2-coil wireless power transfer systems[C], 2014 American Control Conference(ACC), June 4-6, 2014.

[16] Alanson P. Sample, David A. Meyer, Joshua R. Smith, Analysis, experimental results, and range adaptation of magnetically coupled resonators for wireless power transfer, IEEE Trans. Ind. Electron., vol.58, no.2, pp.544-554, Feb. 2011. 